\title{
Ring Arrangement of Active Ranging Sensors for Autonomous Mobile Robot
}

\author{
Soon-cheol Kim ${ }^{1}$, Chan-ho Park ${ }^{2}$ and Soo-yeong $\mathrm{Yi}^{3}$ \\ ${ }^{1,2}$ Department of Electrical and Information Engineering, Seoul National \\ University of Science and Technology, Korea \\ soonchuls@naver.com:cpktph@seoultech.ac.kr \\ ${ }^{3}$ Corresponding author, Department of Electrical and Information Engineering, \\ Seoul National University of Science and Technology, Korea \\ suylee@seoultech.ac.kr
}

\begin{abstract}
Distance measurement is essential for autonomous mobile robot. In this paper, development of a structured-light image based ranging system is addressed. In order to obtain omnidirectional distance data, the ranging sensor units are deployed in ring structure on a mobile robot. The omnidirectional distance information to surrounding objects is useful for making a local distance map and the self-localization of a mobile robot by matching the local map with a given global map. An efficient image processing algorithm, i.e., integration of difference images with structured-light modulation is proposed, which results in robust extraction of the structured-light pixels from a camera image against environmental ambient light. Experiments for the structured-light pixel extraction, distance computation, matching and localization are conducted to verify the performance of the proposed ranging system.
\end{abstract}

Keywords: Active ranging, Mobile robot, Matching, Structured-light

\section{Introduction}

In order to achieve self-localization and autonomous navigation for a mobile robot, a distance measurement system is prerequisite to obtain spatial map of the robot's environment. Among the many kinds of ranging sensors developed so far, the structuredlight image based sensor has many advantages over the others: efficient computation in image processing, robustness against ambient light and cost-effectiveness [1].

The structured-light imaging system projects a light of a distinct frequency in a particularly structured pattern onto environmental object and captures the reflected structured-light by a conventional camera. The distance to the object is computed based on the distortion of the structured pattern which is a function of the distance. Many results are available that use this method. M. Altschuler et al. proposed a structured-light imaging system with $\mathrm{MxN}$ dot matrix laser beam pattern to reconstruct 3D shape of an object [2]. In [3], A. Blake et al. solved the epipolar geometry and correspondence problem of multiple stripes of structured-light pattern.

The bulky laser equipments and the image processing time has discouraged the use of the structured-light image based method in the past, however recent advancements in semi-conductor laser equipments and fast processors have made this system more viable and economical. Thus, the structured-light image based ranging system has been used for mobile robots [4-6].

On the other hand, the structured-light imaging system is also widely used for 3D shape reconstruction by dense structured-light pattern through spatial-temporal codification [7-8]. Survey on this area is well-described in [7]. Y. Oike developed a smart 
integrated circuit image sensor for the structured-light based 3D shape reconstruction [9]. Since 3D information of an object is necessary for robotic manipulation, the dense and multiple structured-light patterns should be employed for 3D shape reconstruction of the object. On the contrary, 2D distance information to an object is enough for autonomous navigation of a mobile robot on flat ground. The 3D shape reconstruction demands cumbersome projector and camera devices and loses the realtime since it spends much computation time in general.

It is clear that the multi-directional distance is more useful for free motion of a mobile robot in cluttered environment. The panoramic structured-light imaging systems were proposed in [10-12] to acquire omnidirectional distances. The omnidirectional distance data can be used not only for deciding the heading direction but also for the selflocalization and autonomous navigation by making a local object map and matching with a given global map.

In this paper, a structured-light image based ranging sensor module is developed with an embedded image processor and a laser diode generating stripe structured-light. The ranging sensor units are deployed in ring structure on a mobile robot to obtain the omnidirectional distances. Each ranging module processes structured-light image to extract distance information and transmits the distance data to main controller of the mobile robot. The main controller performs the self-localization by estimation of robot's posture (position and heading angle) through matching the omnidirectional distance data with given global object map. This paper is organized as follows: Section 2 describes the structured-light image based ranging sensor and the distance measurement. Section 3 addresses the matching and the self-localization algorithm using the omnidirectional distance data. Experimental results are presented in Section 4 and conclusion is in Section 5 .

\section{Development of Structured-Light Image Based Ranging System}

The structured-light image based ranging sensor consists of a camera and a structuredlight source as shown in Figure 1. In order to get horizontal distances to objects under the assumption of robot motion on flat ground, a horizontal sheet of laser structured-light is used in this paper. One easy way to remove background image and extract only the structured-light pattern is to use an optical band-pass filter that only transmits the particular frequency of the structured-light, but a sharp band-pass filter has a high cost. Another method to extract structured-light is to compute the difference of two images with the structured-light modulation: one with the structured-light on and the other without it as illustrated in Figure 2. This technique is commonly used to detect a motion on a static scene. A structured-light modulation circuits are needed to use the imagedifference method.

In order to compensate for the weakened structured-light and make it resistant to the ambient background lights, a special image processing algorithm is needed that emphasize the structured-light. In this paper, a temporal integration method of the difference images is used to selectively enhance the structured-light as in Figure 2. The number of integrations is predetermined as 3 taking the strength of the ambient light into consideration in this paper [13].

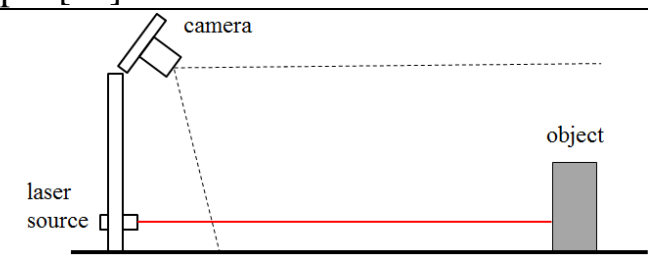

Figure 1. Distance Measurement based on Structured-light Image 


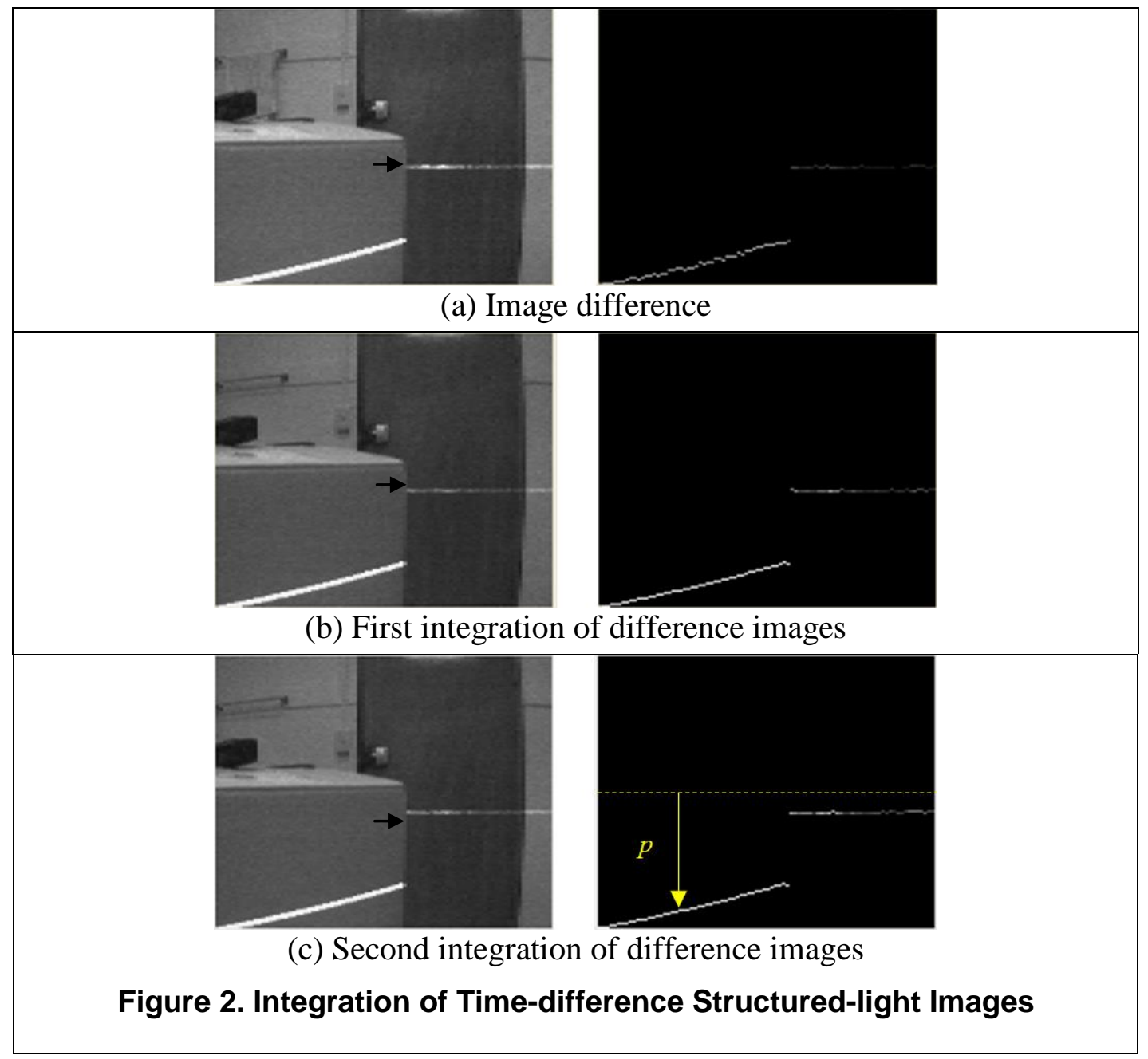

The structured-light pixel distance is denoted as $p$ in Figure 2 (c) from the center line of the image in vertical direction. The measurement angle $\rho$ is given as follows:

$$
\rho=\tan ^{-1}\left(\frac{p}{\lambda}\right)
$$

where $\lambda$ represents the focal length of the camera. From the distance measurement model in Figure 3, the distance to an object, $l$, can be obtained as

$$
l=b \cdot \cot \left\{\theta-\tan ^{-1}\left(\frac{p}{\lambda}\right)\right\}
$$

where $\theta$ represents camera view angle, and $b$ denotes baseline respectively.

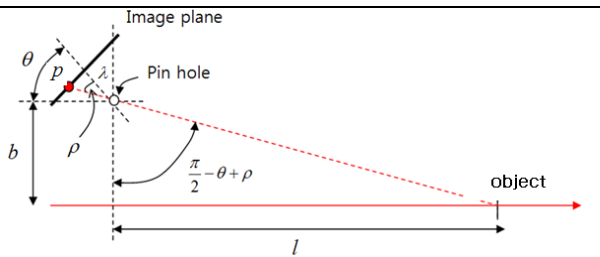

Figure 3. Distance Measurement Model 
The embedded camera CMUcam3 [14] and a 660nm wavelength infrared semiconductor laser are used to develop the structured-light image based ranging sensor module as shown in Figure 4. The embedded processor carries out the whole image processing and transmits only the distance data to the main controller of robot. Figure 5 shows the ring array of the ranging sensor modules attached on a mobile robot which can measures omnidirectional object distances.

\section{Self-localization Using Omnidirectional Distance Map}

\subsection{Local Omnidirectional Distance Map}

Figure 6 shows the structured-light pixel images from the ring array of the ranging sensors in an exemplar environment of a mobile robot shown in Figure 7. In Figure 7 (a), a circled number denotes each camera in the ring array corresponding to each image in Figure 6. From the distance equation (2), it is possible to acquire a local omnidirectional distance map as shown in Figure 7 (b). The local distance map consists of a set of measured points $\left(x_{m}, y_{m}\right)$ represented in the moving coordinates of the mobile robot.

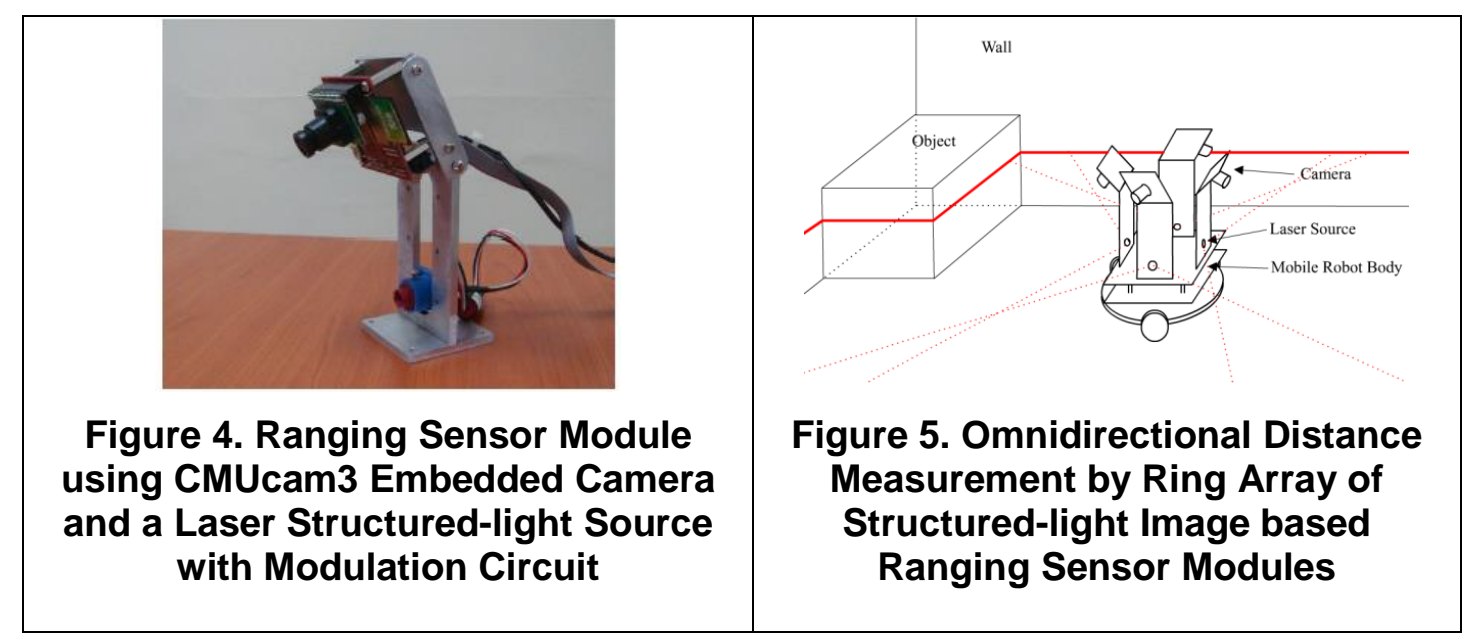

When the present posture of the robot is $\left(\hat{x}_{r}, \hat{y}_{r}, \hat{\theta}_{r}\right)$ in the world coordinates, the measured local distance data in the moving coordinate can be transformed into the world coordinates as follows:

$$
\left[\begin{array}{l}
x_{w} \\
y_{w}
\end{array}\right]=R\left(\hat{\theta}_{r}\right)\left[\begin{array}{l}
x_{m} \\
y_{m}
\end{array}\right]+T\left(\hat{x}_{r}, \hat{y}_{r}\right)
$$

where $R(\theta)$ and $T(x, y)$ represent the rotation and translation respectively as follows:

$$
R(\theta)=\left[\begin{array}{cc}
\cos \theta & -\sin \theta \\
\sin \theta & \cos \theta
\end{array}\right] \quad T(x, y)=\left[\begin{array}{l}
x \\
y
\end{array}\right]
$$

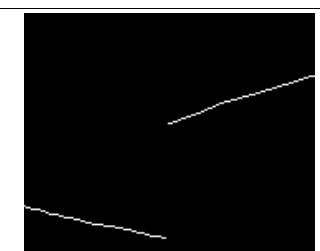

(a) From camera 1

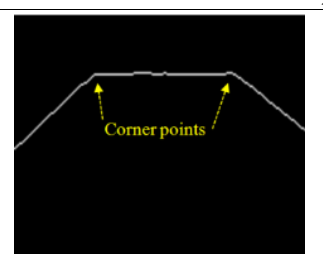

(b) From camera 2

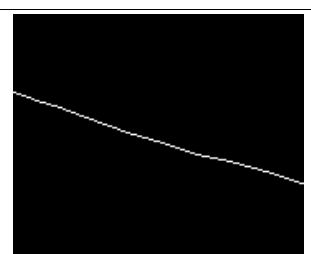

(c) From camera 3

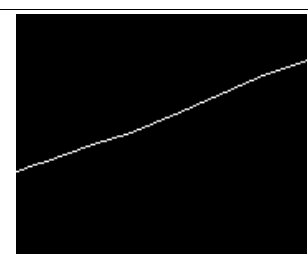

(d) From camera 4

Figure 6. Structured-light Pixel Images from Ranging Sensor Array 


\subsection{Matching for Self-localization}

The self-localization of the mobile robot can be made by matching of the omnidirectional distance map with a given global object map. There have been many researches on the map matching problem. In [15-16], the least-squared error based point matching algorithm was suggested. Since the matching algorithm considered each measured point individually, it requires lots of computations. The omnidirectional distance map in this paper contains many measured point data, thus, the computational burden would be much more severe. In order to improve efficiency in computation, a modified line matching algorithm is presented in this paper: Line segments are obtained from the measured omnidirectional distance map first and match only two end points of a line segment with the global map rather than all measured points.

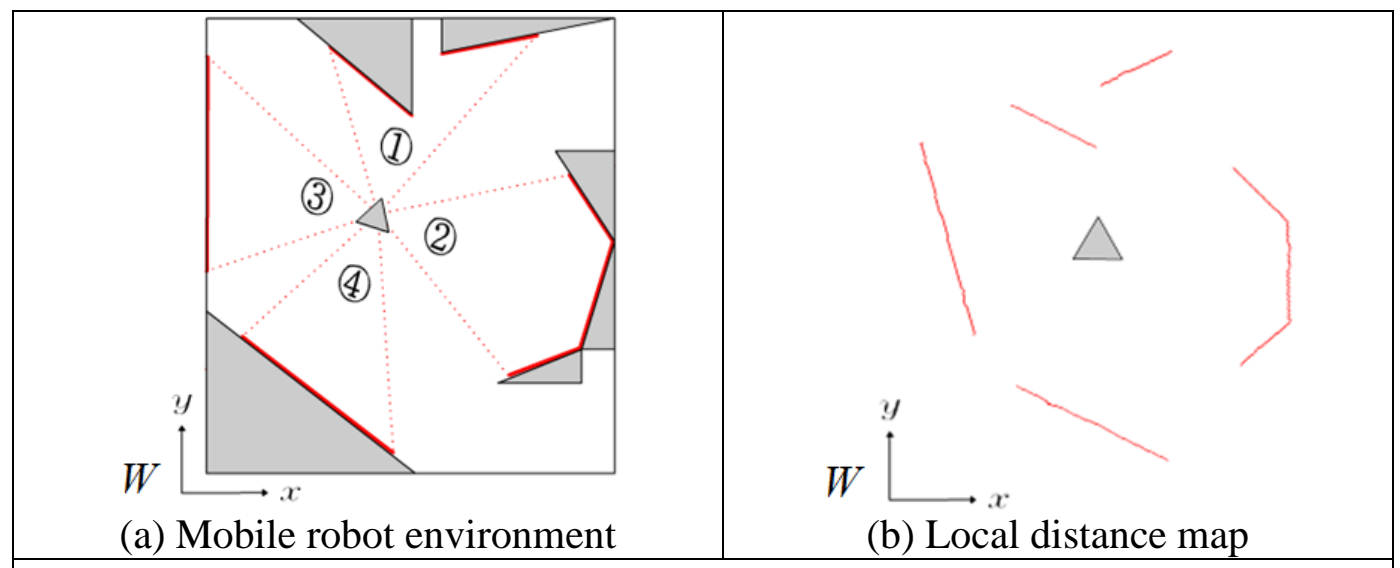

Figure 7. Omnidirectional Distance Data

\subsection{Line Segment from the Measured Distance Data}

In order to represent the measured distance data with several line segments, two end points of a line segment should be determined. In case that there is a discontinuous point in the measured data as in Figure 6 (a), it is simple to determine the end point of a line segment. In the other case that two line segments are connected as in Figure 6 (b), a corner point should be found out as the end point of a line segment. As illustrated in Figure 8, an angle $\theta_{i}$ at $P_{i}$ between the vectors $\vec{r}_{i}=\overrightarrow{P_{i} P_{i-n}}$ and $\vec{f}_{i}=\overrightarrow{P_{i} P_{i+n}}$ are defined as follows:

$$
\theta_{i}=\cos ^{-1}\left(\frac{\vec{r}_{i} \cdot \vec{f}_{i}}{\left\|\vec{r}_{i}\right\|\left\|\vec{f}_{i}\right\|}\right)
$$

where ". denotes the inner product, $P_{i}$ is the $i^{\text {th }}$ pixel position in the measured data, and $P_{i-n}$ and $P_{i+n}$ are the $(i-n)^{t h}$ and the $(i+n)^{t h}$ pixel positions respectively with a fixed interval $n$. The criterion for $P_{i}$ to be a corner point is described as follows:

$$
\theta_{i-n} \geq \theta_{i-(n-1)} \geq \cdots \geq \theta_{i} \text { and } \theta_{i} \leq \theta_{i+1} \leq \cdots \leq \theta_{i+n}
$$

Eq. (6) implies that $\theta_{i}$ is a local minimum within $i-n \leq i \leq i+n$. Figure 9 shows the influence of pixel noise on the angle $\theta_{i}$ in accordance with $n$. When there is not any noise on pixel $P_{i}$, the angle should be $\theta_{i}$ in the figure. If the amount of noise on $P_{i}$ is $\varepsilon$ in $y$ axis, the angle becomes $\theta_{i}^{\prime}$ or $\theta_{i}^{\prime \prime}$ in case of $n=1$ or $n=4$ respectively as shown in the figure. Thus, the influence of pixel noise on the angle become smaller as the interval $n$ is increased. However, if $n$ is set too large, it may cause a loss of corner point in a short line 
segment. Thus, the size of $n$ should be determined by taking the amount of pixel noise into consideration. Figure 10 is the graph of the angle $\theta_{i}$ by applying (5) to the image in Figure 6 (b). From two end points of a line segment, the center of the line segment and the number of measured data points on the line segment can also be obtained.
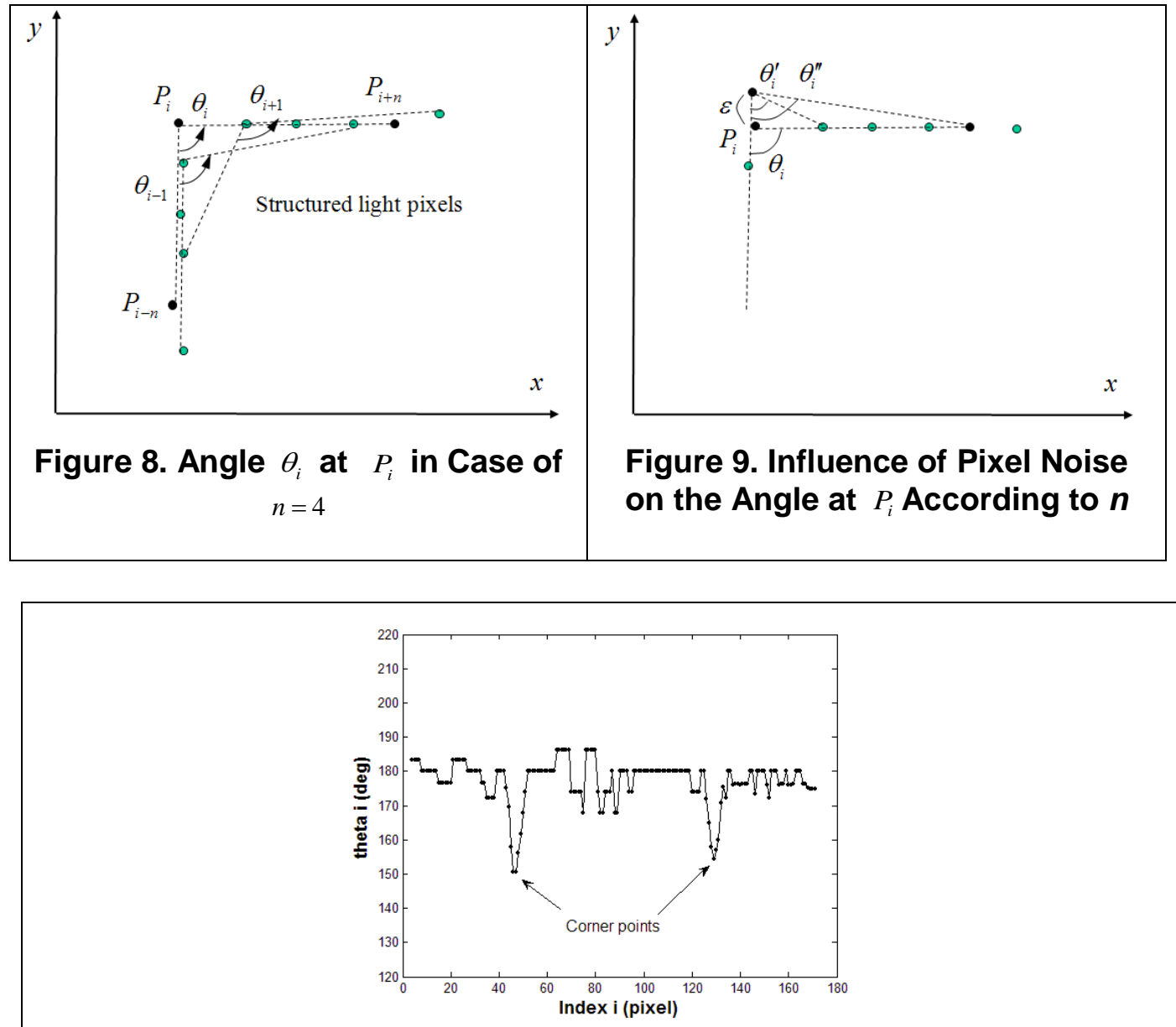

Figure 10: Angle $\theta_{i}$ with respect to $i$ at $n=4$

\subsection{Matching and the Self-localization}

In Figure 11 (a), $P_{l}^{i}$ and $P_{l}^{e}$ denote two end points of a line segment $l$. The center of the segment is $P_{l}^{c}=\left(P_{l}^{i}+P_{l}^{e}\right) / 2$ and $n_{l}$ is the number of data points on the segment $l$. It is assumed that the global map is modeled by the polygonal objects. The model line in Figure 11 represents a side line of the polygonal object.

As explained in Figure 11, the nearest model line is chosen as a target for each measured line segment $l$. To choose the target line, the shortest distance between a model line and the center of a line segment is used. The target line is described as

$$
P \cdot \mathbf{u}_{l}=r_{l}
$$

where $P$ is a point on the target line, $\mathbf{u}_{l}$ is the unit normal vector, and $r_{l}$ is a certain real number. 


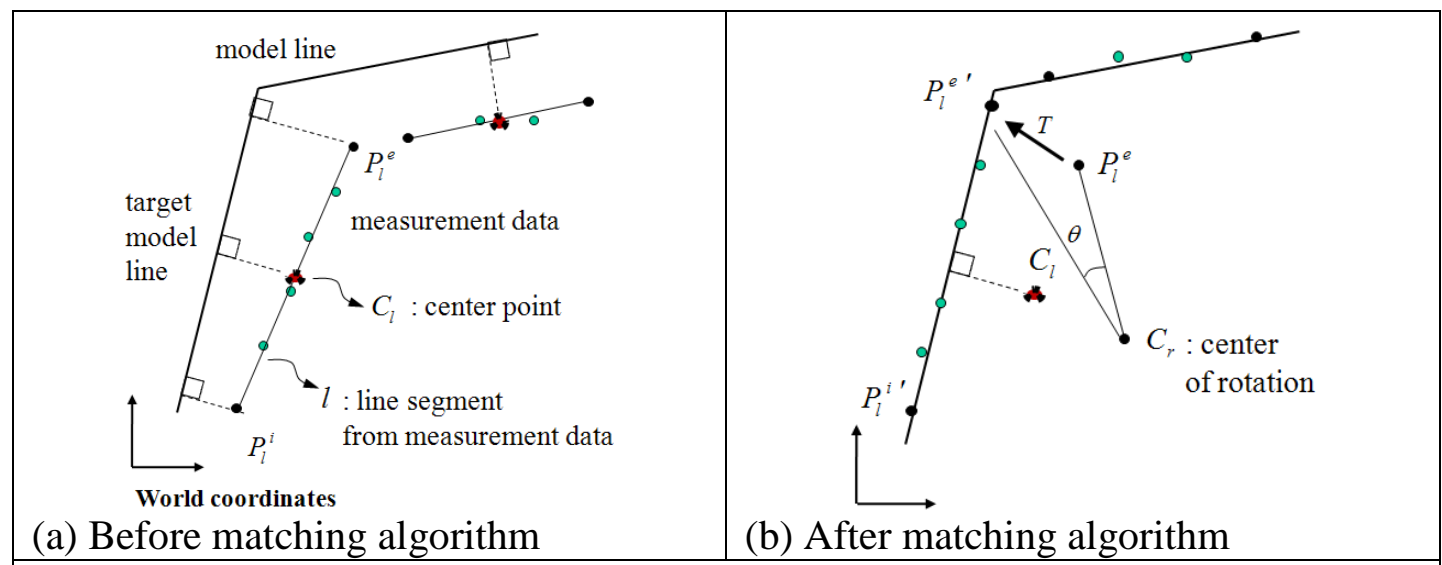

Figure 11. Matching Algorithm

Rotation by $\Delta \theta$ and translation by $(\Delta x, \Delta y)$ with respect to the reference position $C_{r}$ of the robot make the end points of the segment $l$ as

$$
P_{l}^{\prime}=R(\Delta \theta)\left(P_{l}-C_{r}\right)+C_{r}+T(\Delta x, \Delta y)
$$

where $P_{l}$ and $P_{l}^{\prime}$ represent an end point before and after the transformation respectively. The squared distance between the transformed end point and the target line (7) is defined as the matching error as follows:

$$
\begin{aligned}
s_{l}= & {\left[\left\{R(\Delta \theta)\left(P_{l}^{i}-C_{r}\right)+C_{r}+T(\Delta x, \Delta y)\right\} \cdot \mathbf{u}_{l}-r_{l}\right]^{2} } \\
& +\left[\left\{R(\Delta \theta)\left(P_{l}^{e}-C_{r}\right)+C_{r}+T(\Delta x, \Delta y)\right\} \cdot \mathbf{u}_{l}-r_{l}\right]^{2}
\end{aligned}
$$

where $P_{l}^{i}$ and $P_{l}^{e}$ are the two end points of the line segment. Then, the total matching error is represented by sum of the weighted matching errors of all line segments:

$$
\begin{aligned}
S= & \sum_{l} n_{l} s_{l} \\
= & \sum_{l} n_{l}\left[\left\{R(\Delta \theta)\left(P_{l}^{i}-C_{r}\right)+C_{r}+T(\Delta x, \Delta y)\right\} \cdot \mathbf{u}_{l}-r_{l}\right]^{2} \\
& +\sum_{l} n_{l}\left[\left\{R(\Delta \theta)\left(P_{l}^{e}-C_{r}\right)+C_{r}+T(\Delta x, \Delta y)\right\} \cdot \mathbf{u}_{l}-r_{l}\right]^{2}
\end{aligned}
$$

The weight $n_{l}$ is the number of all data points on a line segment $l$. In order to get the amount of rotation $\Delta \theta$ and translation $(\Delta x, \Delta y)$ that minimizes the total matching error (10) by the least-square method, the rotational matrix $R(\Delta \theta)$ is linearized as follows [16]:

$$
R(\Delta \theta)=\left[\begin{array}{cc}
\cos (\Delta \theta) & -\sin (\Delta \theta) \\
\sin (\Delta \theta) & \cos (\Delta \theta)
\end{array}\right] \approx\left[\begin{array}{cc}
1 & -\Delta \theta \\
\Delta \theta & 1
\end{array}\right]
$$

By inserting (11) into (10) and taking a derivative with respect to $\Delta \theta$ and $(\Delta x, \Delta y)$, it is possible to get the amount of translation and rotation that minimizes the total matching error as follows:

$$
\left[\begin{array}{c}
\Delta x \\
\Delta y \\
\Delta \theta
\end{array}\right]=\left[\begin{array}{ll}
A_{2 \times 2} & B_{2 \times 1} \\
C_{1 \times 2} & D_{1 \times 1}
\end{array}\right]^{-1} \cdot\left[\begin{array}{c}
E_{2 \times 1} \\
F_{1 \times 1}
\end{array}\right]
$$

where 


$$
\begin{aligned}
& A_{2 \times 2}=\sum_{l} n_{l} \mathbf{u}_{l} \mathbf{u}_{l}^{t} \\
& B_{2 \times 1}=\sum_{l} n_{l}\left\{M\left(P_{l}-C_{r}\right) \cdot \mathbf{u}_{l}\right\} \mathbf{u}_{l} \\
& C_{1 \times 2}=B_{2 \times 1}^{t} \\
& D_{1 \times 1}=\sum_{l} n_{l}\left\{M\left(P_{l}-C_{r}\right) \cdot \mathbf{u}_{l}\right\}^{2} \\
& E_{2 \times 1}=\sum_{l} n_{l}\left(r_{l}-P_{l} \cdot \mathbf{u}_{l}\right) \mathbf{u}_{l} \\
& F_{2 \times 1}=\sum_{l} n_{l}\left(r_{l}-P_{l} \cdot \mathbf{u}_{l}\right)\left\{M\left(P_{l}-C_{r}\right) \cdot \mathbf{u}_{l}\right\}
\end{aligned}
$$

In (13), $M$ is given by

$$
M=\left[\begin{array}{cc}
0 & -1 \\
1 & 0
\end{array}\right]
$$

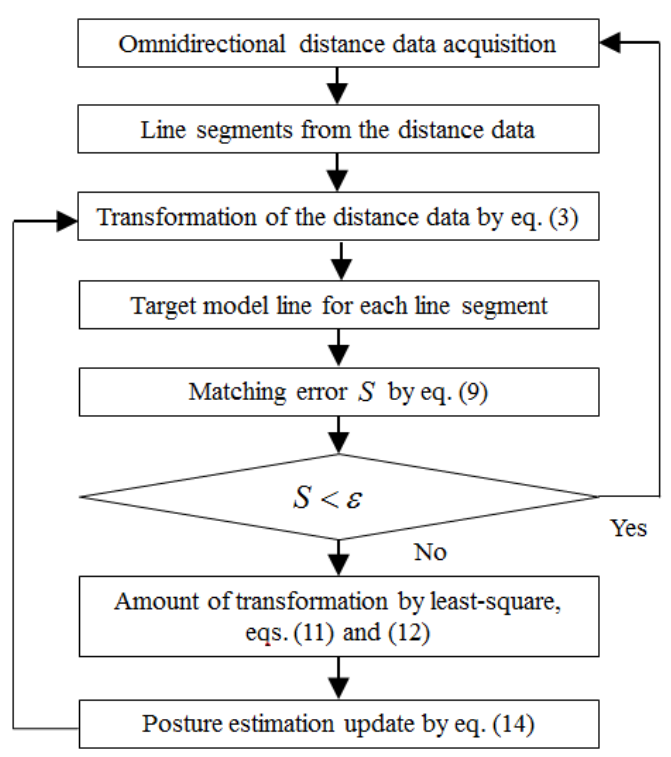

Figure 12. Matching Process

When the amount of transformation $(\Delta x, \Delta y, \Delta \theta)$ is obtained from (12), the estimated posture $\left(\hat{x}_{r}, \hat{y}_{r}, \hat{\theta}_{r}\right)$ should be updated as follows :

$$
\left(\hat{x}_{r}, \hat{y}_{r}, \hat{\theta}_{r}\right) \leftarrow\left(\hat{x}_{r}+\Delta x, \hat{y}_{r}+\Delta y, \hat{\theta}_{r}+\Delta \theta\right)
$$

The matching process from (7) through (15) should be repeated until the matching error (10) becomes smaller than a predefined value. Overall matching process is represented by a flowchart in Figure 12.

\section{Experimental Results}

To verify the performance of the proposed ranging system in this paper, experiments on the self-localization and the autonomous navigation of a mobile robot are conducted.

\subsection{Experimental Environment and Mobile Robot}

As shown in Figure 13 (a), several polygonal objects are placed in the environment of a mobile robot. Figure 13 (b) shows the mobile robot and the ranging system consisting of 4 structured-light image based embedded sensors. 


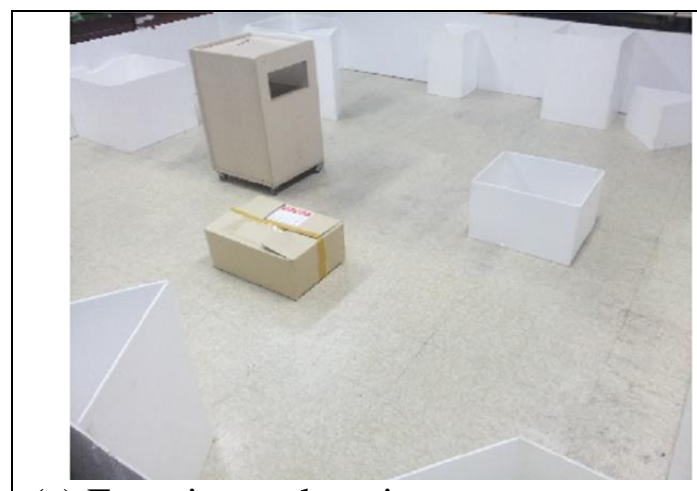

(a) Experimental environment
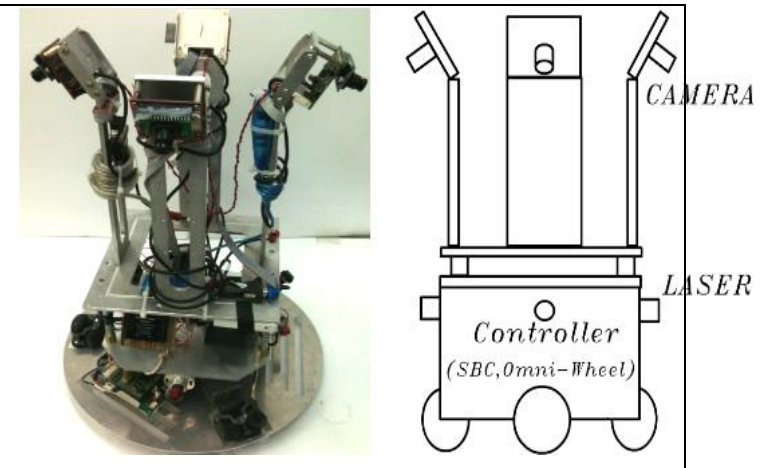

(b) Mobile robot and ranging system

Figure 13. Experimental Environment and Mobile Robot

\subsection{Acquisition of Distance Data}

Image acquisition rate of CMUcam3 is 26 FPS(Frames Per Second). The ranging frequency is around $4 \mathrm{~Hz}$ since 6 image frames are needed for the sequential integration of difference images: Two image frames to get difference image with the modulated structured-light and three difference images for the sequential integration to improve detectability for the structured-light pixels in the image.

The maximum measurable distance of the ranging sensor module in this paper is about $1,200 \mathrm{~mm}$. As explained in Figure 3, the measurable distance depends on the camera view angle and the baseline. Figure 14 shows the actual distance in accordance with the measured pixel distance, $p$ in (2) and the distance measurement error. From the figure, the approximate distance measurement error is within $\pm 10 \mathrm{~mm}$ at the distance range of $1,200 \mathrm{~mm}$.

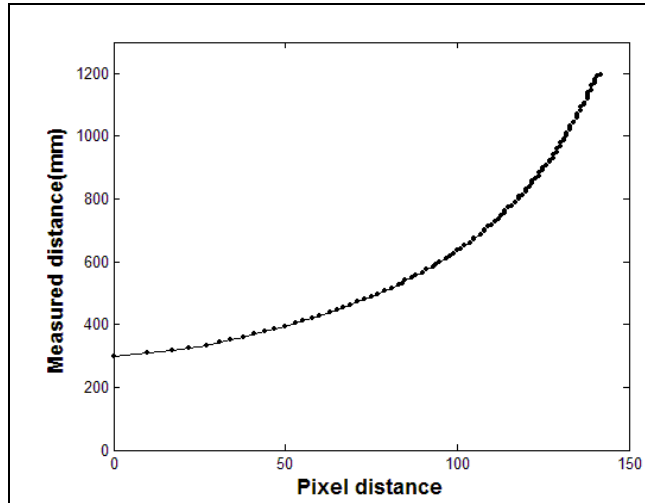

(a) Measured distance

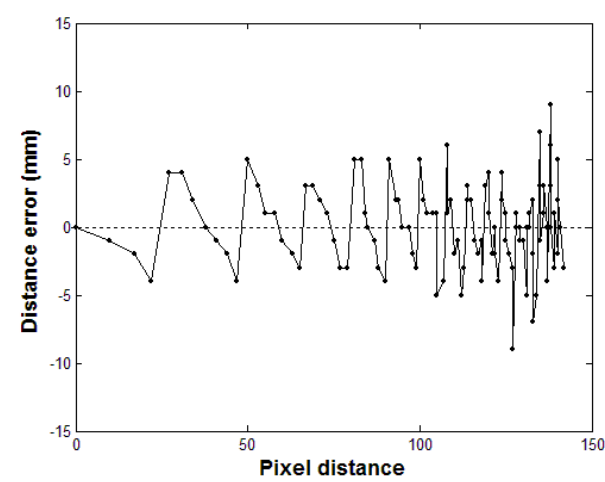

(b) Distance measurement error

Figure 14. Measured Distance and Measurement Error

\section{Result of Experiments}

Figure 15 shows the result of the map matching and the self-localization algorithm. The omnidirectional distance data measured at an unknown robot posture is depicted by red line segments in Figure 15 (a) and the resultant robot posture after the matching is represented by a dark triangle in Figure 15 (b). The amount of transformation to update the robot posture is $(\Delta x, \Delta y, \Delta \theta)=\left(630.0,320.0,16.54^{\circ}\right)$ from the matching algorithm. 


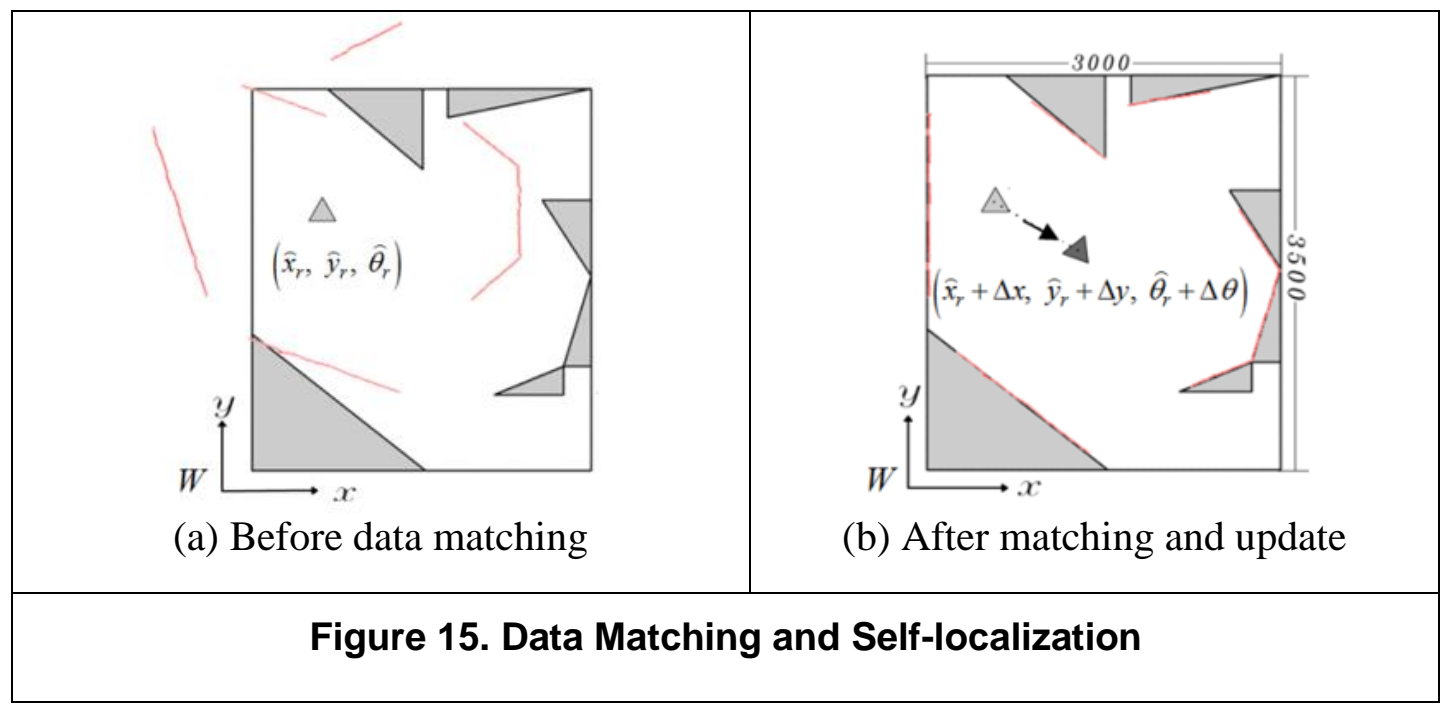

Figure 16 shows the result of the autonomous navigation of the robot with the real-time omnidirectional distance measurement and the localization through the matching algorithm. The black polygons represent the global object map. The line from the start to the goal is the given desired path for the robot and the series of the small triangles represent the actual robot positions with heading angle. As described in (11), the matching algorithm appoximates the rotational matrix in linearization. Size of the matching error that can be converged by the approximated matching algorithm might be dependant on an occasion in general. Using a computer simulation, it is shown in this paper that the approximated matching algorithm converges within $45^{\circ}$ of rotational angle error.

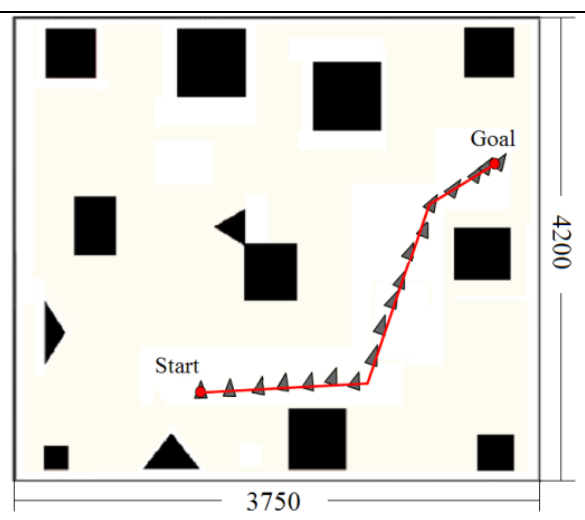

Figure 16. Result of Tracking Control for Mobile Robot

\section{Conclusions}

The ring array of the structured-light image based ranging sensors proposed in this paper is able to obtain omnidirectional distance that makes to achieve fast localization for autonomous navigation of a mobile robot. A compact camera with an embedded processor used for the ranging sensor in this paper sends only final distance data to the main controller of the robot, thus lowering its computational burden.

It is required a matching between the omnidirectional distance data from the proposed ranging sensors and a given global object map for the self-localization of the mobile 
robot. A least-squared error based algorithm is developed in this paper to associate between line segments extracted from the measured omnidirectional distance data and polygonal model of the global object map. Since the matching algorithm in this paper used only two end points of a line segment to associate with an edge of the polygonal model, it is efficient in computation than a conventional point to point matching algorithm.

The main contributions of this paper are summarized as:

- Cost-effective embedded ranging sensor module

- Ring array of the ranging sensors for omnidirectional distance measurement

- Robustness against ambient illumination by sequential integration of difference images with modulated structured-light

- Efficient computation in map-matching by line-line matching

The proposed ring array of the structured-light image based ranging sensor and the matching algorithm in this paper were verified through experiments.

\section{Acknowledgements}

[1] This research was supported by Basic Science Research Program through the National Research Foundation of Korea (NRF) funded by the Ministry of Education, Science and Technology (2011-0009113)

\section{References}

[2] R. Jain, R. Kasturi, and B. Schunck, (1995), Machine Vision, McGraw-Hill.

[3] M. Altschuler, B. Altschuler, and J. Taboceda, "Laser electro-optic system for rapid three-dimensional topographic mapping of surfaces”, Optical Engineering, vol. 20, (1981), pp. 953-961.

[4] A. Blake, D. McCowen, H. Lo, and P. Lindsey, "Trinocular Active Range-Sensing," IEEE Tr. On Pattern Analysis and Machine Intelligence, vol. 15, no. 5, (1993) May, pp. 477-483.

[5] A. Escalera, L. Moreno, M. Salichs, and J. Armingol, "Continuous mobile robot localization by using structured light and a geometric map," International Journal of Systems Science, vol. 27, no. 8, (1996), pp. 771-782.

[6] J. Diebel, K. Reutersward, S. Thrun, J. Davis, and R. Gupta, "Simultaneous Localization and Mapping with Active Stereo Vision," Proc. of IEEE/RSJ International Conference on Intelligent Robots and Systems, Sept. Sendai, Japan, (2004), pp. 3436-3443.

[7] M. Jung, H. Myung, S. Hong, D. Park, H. Lee, and S. Bang, "Structured Light 2D Range Finder for Simultaneous Localization and Map-building (SLAM) in Home Environments," Proc. of 2004 International Symposium on Micro-Nanomechatronics and Human Science, (2004), pp. 371-376.

[8] J. Salvi, J. Pages, and J. Batlle, "Pattern codification strategies in structured light system," Pattern Recognition, vol. 37, no. 4, (2004), pp. 827-849.

[9] M. Kim, and H. Cho, "An active trinocular vision system of sensing indoor navigation environment for mobile robots," Sensors and Actuators A., vol. 125, (2006), pp. 192-209.

[10] Y. Oike, "Smart Image Sensors and Associative Engines for Three Dimensional Image Capture", Ph.D. Thesis, Univ. of Tokyo, (2004).

[11] S. Yi, B. Choi and N. Ahuja, "Real-time omni-directioanl distance measurement with active panoramic vision", Int'l Journal of Control, Automation and Systems, vol. 5, no. 2, (2007), pp. 184-191.

[12] R. Orghidan, E. Mouaddib and J. Salvi, "A Computer Vision Sensor for Panoramic Depth Perception," Proc. of the 2nd Iberian Conf. on Pattern Recognition and Image Analysis, Estoril, Portugal, Lecture Notes in Computer Science, vo. 3522, (2005), pp. 153-160.

[13] I. Joung, and H. Cho, "An active omnidirectional range sensor for mobile robot navigation," Control Engineering Practice, vol. 6, no. 3, (1988), pp. 385-393.

[14] S. Yi, J. Suh, Y. Hong, and D. Hwang, "Active Ranging System Based on Structured Laser Light Image," Proc. of SICE, Taiwan, (2010), pp. 747-752.

[15] http://www.cmucam.org

[16] I. Cox, "Blanche-an experiment in guidance and navigation of an autonomous robot vehicle," IEEE Transactions on Robotics and Automation, vol. 7, no. 2, (1991), pp. 193-204.

[17] I. Cox and J. Kruskal, "On the Congruence of Noisy Images to Line Segment Models," Proc. of Int'l Conf. on Computer Vision, (1988), pp. 252-258. 


\section{Authors}

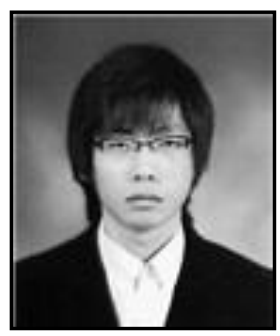

Soon-cheol Kim, he received the B.S. degree in Electrical and Information Engineering from Seoul National University of Technology, Korea. He is now M.S course in Electrical and Information Engineering from Seoul National University of Technology, Korea. His primary research interest is in the area of robot vision, and mobile robot

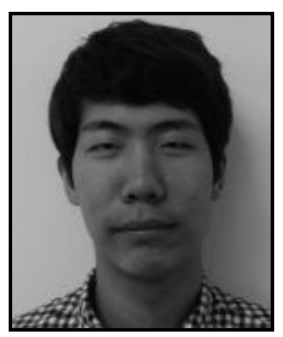

Chan-ho Park, He is now B.S course in Electrical and Information Engineering from Seoul National University of Technology, Korea. His primary research interest is in the area of robot vision, and mobile robot

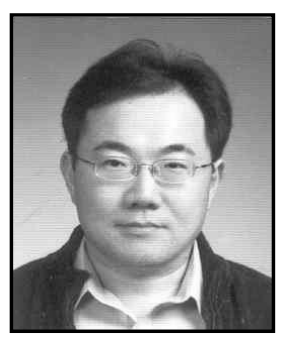

Soo-yeong Yi, he received the M.S. and Ph.D. degrees in Electrical Engineering from Korea Advanced Institute of Science and Technology in 1990 and 1994 respectively. During 1995-1999, he stayed in Human Robot Research Center in Korea Institute of Science and Technology as a senior researcher. He was a professor in the Div. of Electronics and Information Engineering, Chonbuk National University, Korea from Sept. 1999 to Feb. 2007. He also was a post doctorial researcher in the Dept. of Computer Science, University of Southern California, Los Angeles in 1997 and a visiting researcher in the Dept. of Electrical and Computer Engineering, University of Illinois at Urbana-Champaign in 2005. He is now with the Dept. of Electrical and Information Engineering in Seoul National University of Technology, Korea. His primary research interest is in the area of service robot, robot vision, and intelligent control theory. 